
échanges littéraires et culturels (XIVe-XV|e siècle)

\title{
Impressions italiennes : imprimeurs, auteurs et livres italiens à Lyon au XVI siècle
}

Impressioni italiane: stampatori, autori e libri italiani a Lione nel Cinquecento Italian Impressions: Italian Printers, Authors and Books in Lyon in the 16th Century

Ilaria Andreoli

\section{CpenEdition}

\section{Journals}

Édition électronique

URL : http://journals.openedition.org/cei/5167

DOI : $10.4000 /$ cei.5167

ISSN : 2260-779X

Éditeur

UGA Éditions/Université Grenoble Alpes

Édition imprimée

ISBN : 978-2-37747-063-1

ISSN : 1770-9571

Référence électronique

Ilaria Andreoli, «Impressions italiennes : imprimeurs, auteurs et livres italiens à Lyon au xvl ${ }^{e}$ siècle », Cahiers d'études italiennes [En ligne], 27 | 2018, mis en ligne le 30 septembre 2018, consulté le 27 mars 2021. URL : http://journals.openedition.org/cei/5167 ; DOI : https://doi.org/10.4000/cei.5167

Ce document a été généré automatiquement le 27 mars 2021. 


\title{
Impressions italiennes : imprimeurs, auteurs et livres italiens à Lyon au $\mathrm{XVI}^{\mathrm{e}}$ siècle
}

\author{
Impressioni italiane: stampatori, autori e libri italiani a Lione nel Cinquecento \\ Italian Impressions: Italian Printers, Authors and Books in Lyon in the \\ 16th Century
}

Ilaria Andreoli

1 «Lyon l'Italienne » : l'expression rappelle d'abord l'apport irremplaçable de l'Italie, de ses marchands et de ses banquiers au développement de Lyon du début du $\mathrm{XVI}^{\mathrm{e}}$ siècle jusqu'aux crises répétées des guerres de Religion ${ }^{1}$. De toutes les colonies marchandes étrangères, l'Italienne est la plus nombreuse et la plus active, et Lyon fut, on le sait, un des plus brillants foyers de l'humanisme et de la Renaissance en France ${ }^{2}$. Ce qui est plus difficile à préciser, c'est ce que cet éclat doit à l'influence directe de la Péninsule et ce que lui apporte une réélaboration locale des nouveautés venues d'au-delà des Alpes. Dans la Renaissance lyonnaise, tout ce qui est italien n'est pas dû à des Italiens mais, souvent, à des Lyonnais « italianisants ».

2 Mon point d'observation privilégié sera le Lyon de l'imprimerie, afin d'évaluer la présence italienne dans les ateliers d'imprimeurs, la réception des produits éditoriaux italiens - qu'ils soient dûs à des auteurs italiens, à des imprimeurs italiens, ou publiés en langue italienne-, et le rôle que jouèrent certains imprimeurs lyonnais, particulièrement tournés vers la Péninsule, dans les échanges culturels italo-français.

\section{Des hommes et des livres}

3 Au début du xvI siècle, Lyon, en pleine croissance depuis 1470, n'est pas encore la grande ville qu'elle deviendra pendant le règne de Francois $\mathrm{I}^{\mathrm{er}}$. Sans institution culturelle prestigieuse - elle ne possède pas d'université -, sans parlement, elle ne bénéficie pas d'un statut politique particulier, bien que les ordonnances de Louis XII 
aient favorisé les foires locales pour contrer le monopole commercial des Genevois ${ }^{3}$. Alors que les premières tentatives d'imprimerie à Lyon datent, comme à Venise, du dernier quart du Xve siècle, avec Barthélemy Buyer ${ }^{4}$, la communauté italienne de Lyon se caractérise dès cette période par un haut niveau d'alphabétisation et des revenus élevés, ce qui entraîne la présence d'un lectorat pour les ouvrages en italien comme la disponibilité de capitaux permettant l'installation d'ateliers typographiques et de librairies. Outre ces aspects économiques, l'essor de l'industrie typographique italienne s'explique à la fois par l'éloignement de Lyon vis-à-vis de la Sorbonne et son ouverture aux nouveautés européennes, et, après les revirements politiques des premières guerres d'Italie, par un afflux d'humanistes toscans fuorusciti, qui travaillent pour les libraires.

Dans le panorama éditorial lyonnais, les Italiens s'insèrent donc à différents niveaux. Ils sont fournisseurs de papier ou de caractères, travailleurs qualifiés ou apprentis, transporteurs, libraires dépositaires ou simples courtiers, prêteurs aussi pour des montants parfois importants. Les premiers typographes italiens arrivent directement depuis la Péninsule, souvent du Piémont et de Venise, puis la presque totalité des grandes entreprises éditoriales italiennes s'installe entre la fin $\mathrm{du} \mathrm{xv}^{\mathrm{e}}$ siècle et 1530 (Giunta, Gabiano, Portonariis, Pullon, Sacconi...) et leurs activités prospèrent jusqu'en 1550. Une grande partie provient du Piémont tandis que d'autres, comme les Giunta, sont toscans et ouvrent la voie, dans les années 1550, aux Tinghi et aux Honorat ${ }^{5}$.

\section{Le lobby piémontais}

5 De la région frontalière (Asti, Romano Canavese et surtout la petite ville de Trino, près de Vercelli) sont originaires les Sacconi (Sacon), les Gabiano et les Portonariis et tant d'autres familles qui ont joué un rôle de première importance dans l'histoire du livre italien à Lyon. Ils ont aussi su créer un axe Venise-Lyon, par Trino et le Piémont, sur lequel circulent les livres, souvent interdits, les gens du livre, et d'autres produits comme les épices et la soie ${ }^{6}$. Cette diaspora de familles d'imprimeurs piémontais s'explique par des raisons de caractère géographique et commercial, ainsi que par l'espoir de trouver au-delà des Alpes un milieu libre et ouvert, notamment sur le plan religieux. L'«intraprendenza» des imprimeurs piémontais, et la qualité de leurs ouvrages, ont contribué au succès européen d'un groupe extrêmement solidaire, lié par son appartenance à la "nation italienne » tout entière comme à un véritable lobby piémontais qui sait interagir d'un lieu à l'autre, déplacer hommes et livres, et fonder des succursales gérées pour la plupart par des membres de la famille ou par des hommes de confiance qui deviendront par la suite imprimeurs à leur tour (les Portonariis, par exemple, sont d'abord au service des Gabiano). Nombreuses sont les collaborations avec des imprimeurs-libraires locaux et, à l'occasion de projets éditoriaux conjoints, avec ceux qui sont restés ou sont revenus dans la Péninsule. Comme l'a fait remarquer Rosanna Gorris Camos, la belle devise de Pullon da Trino: «Distantia loci non separat amicitiam» est emblématique de l'unité de la mouvance piémontaise entre France et Italie ${ }^{7}$.

6 Leurs débuts sont liés à la contrefaçon. Les Gabiano n'hésitent pas à se lancer dans la reproduction des éditions aldines: textes classiques, mais aussi Dante et Pétrarque, évidemment sans la marque à l'ancre et au dauphin. Il s'agit, en l'occurrence, des 
premiers exemples du caractère italique en France et des premières éditions italiennes imprimées hors d'Italie, mais c'est une initiative éditoriale isolée, délibérément frauduleuse et tournée vers le seul marché italien ${ }^{8}$. Les principaux domaines d'activité de ces imprimeurs sont plutôt l'édition de volumineux ouvrages de référence en latin, surtout en droit et en médecine - leurs collections de célèbres médecins milanais, bolonais ou ferrarais, constitueront dans les années à venir l'essentiel de la production italienne à Lyon -, et celle d'ouvrages religieux. Jacques Sacon imprime aussi à partir de 1506 quinze éditions bibliques, dont deux en français, et une, aux frais d'Anton Koberger, illustrée avec un curieux mélange d'illustrations allemandes et de copies de bois bibliques vénitiens ${ }^{9}$. En revanche, ils montrent un éloignement durable pour le livre humaniste, pour la philologie, la littérature, pour le livre illustré, pour les romans de chevalerie et les livres en langue vernaculaire, tout un monde auquel cette première génération est totalement étrangère. Les Piémontais s'associent en "compagnies" pour l'impression et la vente d'éditions destinées au marché européen et spécialement italien, avec lequel ils entretiennent des contacts constants. En outre, l'échange de livres entre compatriotes établis dans des lieux différents permet d'augmenter l'assortiment des associés sans nécessiter d'investissement en capital. L'aspect physique du livre lui-même confirme l'hypothèse d'une production des libraires italiens conçue par rapport au marché français, avec l'intention d'attacher une « aura » française à leurs produits grâce à des marques typographiques inspirées de celles de Lyon et de Paris. Et ils font fortune : Luxembourg Gabiano accède à la noblesse par l'achat d'une seigneurie, et les Portonariis, à la tête d'un puissant réseau commercial qui, de Trino et Lyon, s'étend à Venise, Salamanque et Saragosse, renforcent leur ancrage lyonnais par le mariage de Marguerite, nièce de Vincenzo, qui dispose d'un important patrimoine immobilier, avec Guillaume Rouillé, ancien apprenti des Giolito à Venise et destiné à devenir un grand acteur de la vie du livre lyonnais au milieu du siècle, et celui de Madeleine avec Gaspard Trechsel, natif de Lyon et fils de Jean, imprimeur-libraire d'origine allemande.

\section{Le lys de Florence à Lyon : les Giunta}

7 Dynastie florentine de véritables industriels du livre, présente sur les principales places européennes, les Giunta sont actifs à partir des années $1470^{10}$. Avant 1500, Luca Antonio I émigre à Venise, où il se consacre principalement à l'édition de textes liturgiques, ainsi que ponctuellement à celle de livres de droit, de médecine et de philosophie, choix qui vise à satisfaire un lectorat vaste et stable, présent sur l'ensemble du continent, en s'adressant au clergé et au public savant des universités. En revanche, la succursale de Florence, dirigée par son frère Filippo I, se spécialise dans l'édition des auteurs classiques de la tradition florentine (Dante, Pétrarque, Boccace) et des textes humanistes latins et grecs. Dans la deuxième décennie du nouveau siècle, la génération suivante est envoyée dans les villes et les pays les plus stratégiques de l'espace européen où ils construisent un réseau commercial avec des succursales et des entrepôts à Francfort, Anvers et Paris, tandis qu'en 1514 Giovanni (Juan), le fils de Filippo, s'installe en Espagne, travaillant dans différentes villes. À Lyon, Giacomo (Jacques), neveu de Lucantonio I et Filippo I, signe sa première édition connue en 1519. Son oncle Luca Antonio I, auprès duquel il a fait son apprentissage, l'a pourvu d'un important capital initial qu'il investit dans la librairie - et non dans la typographie, préférant travailler avec les nombreux ateliers dont dispose la ville-, et dans le 
négoce. Son entreprise est couronnée de succès puisqu'en 1537 il peut prêter 50000 livres tournois au cardinal de Tournon qui tente de redresser les finances royales pour poursuivre la guerre contre l'Espagne. L'activité de la branche lyonnaise prospère, jusqu'à la fin du xvI siècle, sur trois générations : après Jacques, qui meurt en 1546, ses filles Jeanne et Jacqueline, et enfin Jean-Baptiste Regnauld, fils de Jeanne. Grâce au capital dont il dispose, Jacques devient membre de la Grande Compagnie pour l'impression d'éditions particulièrement complexes et coûteuses tout en s'associant ponctuellement avec d'autres libraires pour des éditions moins exigeantes. La marque au lys rouge se spécialise dans l'édition en latin d'ouvrages de droit, de théologie et de médecine pour une clientèle universitaire, professionnelle et institutionnelle - excluant presque complètement les éditions en grec, spécialité de la branche florentine, ainsi que de livres liturgiques, spécialité des Giunta de Venise et d'Espagne. En outre, ils publient, aussi longtemps que la censure le permet, de nombreuses bibles latines qui intègrent les corrections et les révisions de la Vulgate, ainsi que la première version latine moderne basée sur le texte hébreu, celle de Sante Pagnini ${ }^{11}$. Les premières éditions sont rares, surtout dans la génération de Jacques - celle de Pagnini est due à une initiative de l'auteur -, Jacques reproduisant la plupart du temps des éditions parues en Italie ou en France avec, parfois, de nouveaux paratextes comme des index fournis qui correspondaient à une demande du marché international. Les Giunta valorisent la science italienne, mais le livre en italien reste une exception dans leur production et, pour autant que les sources conservées nous permettent de l'affirmer, dans leur commerce.

\section{Les imprimeurs et libraires italianisants et le livre (en) italien à Lyon : Sébastien Gryphe, Jean de Tournes et Guillaume Rouillé}

8 Dans ce contexte il n'est pas étonnant qu'un libraire au catalogue très spécifique, Sébastien Gryphe, soit actif à Lyon ${ }^{12}$. Formé à Venise, son catalogue humaniste est unique en Europe, avec des auteurs comme Érasme ${ }^{13}$ et André Alciat, et des classiques accompagnés de commentaires dus aux meilleurs écrivains italiens «modernes»: Lorenzo Valla, Iacopo Sannazaro, Angelo Poliziano, Giulio Cesare Scaligero, Pietro Vettori. Les hommes de lettres du nord de la Péninsule ont identifié en lui un entrepreneur qui peut offrir un espace où exprimer librement des orientations spirituelles déviantes, par exemple celles d'Ortensio Lando ${ }^{14}$. Gryphe est le premier imprimeur à publier en 1532 une œuvre italienne contemporaine, les Opere toscane de Luigi Alamanni ${ }^{15}$, poète très lié à la cour de France. À partir de ce livre, apparaît toute une suite de textes destinés au marché italien (où les Opere sont rééditées l'année suivante) qui affirment le potentiel de Lyon comme centre de production dans une langue moderne étrangère au cours des deux décennies suivantes, ainsi qu'en témoigne la déclaration de Jean de Tournes, démarrant la série de ses éditions italiennes avec le Pétrarque de 1545, qui explique avoir commencé « a pratticar nella casa dil S. Gryphio, $e$ dal principio essere stato un di quelli compositori che s'aiutorno a comporre in su la stampa le divine opere di Messer Luigi Alamanni ", épisode auquel il fait remonter sa passion pour le toscan.

Avec Gryphe, nous voyons apparaître une génération d'imprimeurs et libraires lyonnais italianisants qui se montrent plus italiens que les Italiens, faisant de Lyon le centre 
principal de production du livre en italien - ou des traductions en français d'ouvrages italiens - en dehors de l'Italie ${ }^{16}$. Leurs presses sont d'ailleurs alimentées par une deuxième génération d'Italiens, fuorusciti politiques et religieux ${ }^{17}$. Durant les années 1550-1560, la production lyonnaise en italien répond à la demande de l'importante «nation » italienne de Lyon, mais aussi - et surtout - à celle d'un marché élargi à la Péninsule, voire à l'Europe, en concurrence avec les imprimeurs-libraires italiens euxmêmes. L'expansion du toscan en France aurait eu donc lieu presque simultanément avec l'affirmation généralisée de la littérature vulgaire dans la Péninsule à l'époque du concile de Trente ${ }^{18}$, représentée au premier chef par un autre imprimeur d'origine piémontaise actif à Venise, Gabriele, de la dynastie des Giolito, très liés aux Portonariis, qui impose un nouveau modèle de livre en langue vernaculaire, élégant, innovant mais bon marché ${ }^{19}$.

À Lyon, Jean de Tournes ${ }^{20}$, au tournant des années 1540 , est le premier libraire à porter une attention toute particulière à la littérature italienne ${ }^{21}$. Après avoir repris, en 1542, une traduction en prose de l'Orlando furioso attribuée à Jean Martin (Lyon, Sabon pour Thellusson), il se singularise en 1545 avec l'étonnante édition de Pétrarque dédiée à Maurice Scève, comme le sera celle de Dante deux ans plus tard. À partir de là, la présence des œuvres toscanes est constante : de Tournes promeut, à côté des classiques du Trecento, la traduction d'œuvres clefs du débat spirituel en Italie, comme Il beneficio di Cristo (1545) et la Tranquillità dell'anima d'Isabella Sforza (1546 et 1549), double littéraire d'Ortensio Lando, toujours lié à l'édition lyonnaise, comme en témoigne la première apparition de ses Paradossi chez Jean Pullon da Trino (1543 et 1550) ${ }^{22}$.

11 Il est de plus en plus difficile pour de Tournes de s'opposer à la concurrence de Guillaume Rouillé, marchand-libraire qui, formé auprès des Giolito à Venise, a épousé la nièce de Vincenzo Portonariis et établi des liens privilégiés avec la « nation » italienne dont il est " amatore grande ». Dans son catalogue «à l'escu de Venise », riche de près de 840 éditions, 226 éditions sont en langue vernaculaire, dont 70 en italien ${ }^{23}$. Sa production italienne, essentiellement commerciale, repose sur l'édition et la réédition d'auteurs classiques - les « trois couronnes », Dante, Pétrarque, Boccace - et d'auteurs littéraires modernes (Arioste, Castiglione) et leurs dérivés, ainsi que sur des versions en italien d'ouvrages également disponibles en français, latin ou espagnol : Figures de la Bible et du Nouveau Testament, recueils d'emblèmes, traités d'antiquaires, souvent procurés par des collaborateurs italiens et richement illustrés.

12 Si les éditions italiennes de De Tournes, médiocrement imprimées, offrent des textes très fautifs, Rouillé, qui maîtrise parfaitement l'italien, reprend le projet avec une tout autre compétence: il dispose de petits "caratteri begli», destinés à garantir «il contentamento dei lettori» dans des éditions de grande qualité esthétique et de petit format, «da tenere appresso ». La qualité de ses impressions rivalise avec les meilleures productions transalpines, comme le souligne Jean-Baptiste du Four dans son Épitre à Rigaud de Saint-Marsal dans l'édition du Decamerone de 1555 : «Ardisco dire che le stampe di Lyone potranno da qui innanzi quasi che stare al paragone nella lingua toscana a quelle di Venetia, di Fiorenza et di Roma. » Rouillé bénéficie surtout des compétences d'un riche gentilhomme lettré, Lucantonio Ridolfi, véritable "osservatore della lingua toscana » qui lui procure les textes les mieux établis en associant à ses propres travaux tout un réseau de lecteurs érudits, ce qui lui assure une sorte de «label de qualité »"

Malgré les déclarations en sens contraire qu'on lit dans les dédicaces de Rouillé et De Tournes, il n'est pas concevable que ces titres aient pu se tourner exclusivement 
vers le marché français ${ }^{25}$, ni qu'ils aient été imprimés pour fournir des outils de base aux français qui voulaient apprendre la langue toscane. Dans ce but, il y avait une production spécialisée avec quelques titres à succès imprimés dans des petits formats, faciles à vendre, comme en témoignent les nombreuses réimpressions ${ }^{26}$. Si la première édition de Pétrarque par Rouillé est enrichie d'une «tavola di tutte le sue rime » due à Luc'Antonio Ridolfi, et si les suivantes annoncent toujours dans le titre quelque nouveau paratexte pour attirer l'attention des lecteurs ${ }^{27}$, l'édition du Decamerone publiée en 1555 - étroitement liée aux éditions vénitiennes récentes de Giolito et Valgrisi - s'ouvre sur une préface dans laquelle Rouillé décrit son parcours éditorial et son projet culturel : séduit par la douceur et la gravité de la langue toscane, il a décidé de montrer son amour en s'efforçant

[...] di stampare tutti i più lodati et i più pregiati authori che in quella lingua scritto habbiano, accioché mandandogli in luce il più che mi sarà possibile corretti, in un tempo medesimo alla mia affezione sodisfaccendo, possa giovare anchora a coloro i quali della lingua Thoscana sono et amatori et apparatori. Et fra questi molti senza dubbio ne ha hoggi la Francia, a' quali più che ad altri intendo io di arrecare et commodità et giovamento: essendo certissimo che essi non meno degli altri italiani questa lingua sapere disiderano, et compiutamente apparare la potranno; percioché, quanto ai Thoscani, io tengo per fermo (come da' migliori è stato et creduto et scritto) che se vorranno alcuno studio impiegarci, et non in quella del popolaresco uso fermarsi senza più mai innanzi trapassare, più agevolmente et per più corta via che alcun altro potranno alla cima della perfezione d'essa pervenire. Anzi credo io che in ciò fare molto miglior modo i nostri Franzesi che gl'altri Italiani, et più agevolezza, ci troveranno: perciò che essendo essi in una lingua alla Thoscana et differente et assai lontana nati, non potranno la loro con questa mescolare, ma pura, sì come ella è, da buoni libri apprendendola, vaga et gentile la saperranno poi et ragionare et scrivere ${ }^{28}$.

Il s'agit d'une position «bembesque » au carré selon laquelle les Italiens non toscans sont défavorisés par rapport aux Français, car la ressemblance partielle de leur langue avec le toscan leur en rend l'apprentissage plus laborieux. La dédicace à Catherine de Médicis de la traduction de Gabriel Symeoni du Discorso sulla religione antica de' Romani par Guillaume du Choul (1559) avance même :

La purità et dolcezza della lingua toscana pare che sia di presente [...] salita in tanto pregio che, doppo la greca et la latina, i Toscani medesimi studiandola s'ingegnano ogni giorno di renderla più bella; i letterati stranieri l'ammirano et, come hanno fatto l'Ariosto, il Bembo et il Sennazzaro, ne i loro scritti cercano d'imitarla ${ }^{29}$.

Si donc tous les non Toscans sont étrangers, à commencer par les plus grands écrivains toscans du siècle, le marché péninsulaire est largement comparable au français.

\section{Images d'échange, échanges d'images}

Dans les échanges entre France et Italie, le livre illustré joue un rôle particulièrement important. Bernard Salomon pour Jean de Tournes et Pierre Eskrich, le dessinateur attitré "à l'escu de Venise », et leur graveurs, reproduisent dans un style local très reconnaissable les xylographies des éditions Giolito ou Marcolini, comme pour souligner que cette production transalpine participait du meilleur «made in Italy " en matière d'imprimerie ${ }^{30}$.

17 Mais entre Venise et Lyon l'illustration circule dans les deux sens. Parmi les livres à figures imprimés par de Tournes, et encore plus par Rouillé, certains sont, comme 
l'attestent les privilèges, conçus dès l'origine pour être produits en plusieurs langues, de façon à amortir le coût des bois gravés tout en s'assurant une plus large diffusion. Sortent des presses de Rouillé, en version latine, française et en réduction italienne, les Emblemata d'Alciat (1548 et 1549), La magnifica et triumphale entrata, description de l'entrée d'Henri II à Lyon (1549, en latin et en italien), et son propre Promptuaire des medalles, sorte d'histoire du monde par la numismatique, en latin, français et italien (1555), puis, plus tard (1561), en castillan ${ }^{31}$; et encore, dans les deux langues, le Dialogo delle imprese militari et amorose de Paolo Giovio accompagné du Ragionamento de Lodovico Domenichi (1559), suivant l'édition Giolito de 1557. Le prolifique Gabriele Symeoni $^{32}$ fournit à Rouillé les traductions italiennes des deux Discours sur les antiquités romaines de Guillaume du Choul traduits par Gabriel Symeoni (1555 et 1559), les éditions bilingues de ses propres Dialogo pio et speculativo (1560) et Imprese heroiche e morali (1559 et 1561), et une réduction en quatrains de ces dernières accompagnées par celles de Giovio (Le sententiose imprese, 1560).

S'éloignant temporairement de Rouillé, Gabriel Symeoni, publie toujours en version bilingue chez de Tournes Les illustres observations antiques (1558) et l'année suivante La vita et metamorfoseo d'Ovidio, figuré et abrégé en épigrammes (1559), adaptation toscane d'une œuvre déjà publiée en français et en néerlandais deux ans plus tôt, que rendent précieuse les vignettes de Bernard Salomon qui eurent tant d'importance comme modèles pour les arts décoratifs, en particulier pour la majolique "istoriata " italienne, comme d'ailleurs les illustrations des textes sacrés accompagnés de vers, imprimés tant par Jean de Tournes (Figure del Vecchio e del Nuovo Testamento, 1554, avec un poème de Damiano Maraffi pour chaque image) que par Rouillé (Figure della Bibbia e del Nuovo Testamento, 1564) ${ }^{33}$.

Ces éditions bibliques permettent de contextualiser les trois célèbres éditions du Nuovo Testamento évangélique publiées à Lyon, premier centre d'impression de Bibles en italien après Venise au cours de ces années: la traduction d'Antonio Brucioli chez Rouillé (associé à Guillaume Gazeau) en 1547, en 1549 et 1552 (avec de nouvelles émissions en 1550 et 1553) ; celle de Massimo Teofilo en 1551 (sans adresse, mais chez Jean Frellon) et en 1556 (Jean de Tournes et Guillaume Gazeau) ; et l'anonyme déjà publiée à Genève en 1555 qui, exceptionnellement accompagnée du texte latin, paraîtra en 1558 chez Rouillée ${ }^{34}$.

Pendant les années 1550, nombreux sont en effet les gens du livre italiens qui ont choisi la religion réformée et s'affichent publiquement, tels Barthélemy de Gabiano et Sébastien Honorat, en tant que membres éminents de la communauté protestante ${ }^{35}$. Cette prise de responsabilité publique se fait aux dépens de leurs entreprises: les maisons et les boutiques des marchands sont saccagées et les troubles qui suivent le sac de 1562 amènent la suppression des foires annuelles et l'exode des banquiers italiens qui privilégient des villes plus sûres, comme Genève, des compagnons imprimeurs et des grands libraires. Après la reconquête catholique de 1567, et surtout après la SaintBarthélemy, le marché lyonnais du livre subit une crise profonde qui provoque la dispersion du lectorat italien, tandis que la progressive assimilation linguistique des ressortissants italiens résidant dans la ville depuis des générations limite la demande d'ouvrages dans leur langue maternelle.

21 Ainsi se clôt un grand cycle séculaire de présence italienne dans la vie du livre lyonnais. Lyon n'est plus « l'Italienne». 


\section{BIBLIOGRAPHIE}

AlBonico Simone, «Librai italiani a Lione 1540-1580 », Nuova Rivista di Letteratura italiana, vol. 3, $\mathrm{n}^{\mathrm{o}} 1,2000$, p. 203-217.

ANDREOLI Ilaria, «Lyon, nom \& marque civile. Qui sème aussi des bons livres l'usage : Lyon dans le réseau éditorial européen ( $\mathrm{xv}^{\mathrm{e}}-\mathrm{XVI}{ }^{\mathrm{e}}$ siècle) », dans J.-L. Gaulin et S. Rau (éds), Lyon vu/e d'ailleurs (1245-1800) : échanges, compétitions et perceptions, Lyon, Presses universitaires de Lyon, 2009, p. $109-140$.

ANDREOLI Ilaria, «Échanges d'images, images d'échanges : le livre illustré lyonnais à la Renaissance ", dans L. Virassamynaïken (dir.), Lyon Renaissance. Arts et Humanisme, catalogue de l'exposition (Lyon, Musée des Beaux-Arts, 2015), Lyon-Paris, MBA-Somogy, 2015, p. 62-67. ANDREOLI Ilaria, « Un "Lyonnais italianisant" : les éditions italiennes illustrées de Guillaume Rouillé », dans C. Lastraioli (éd.), « Poco a poco ». L'apport de l'édition italienne dans la culture francophone, Actes du $60^{\mathrm{e}}$ colloque international d'études humanistes du Centre d'études supérieures de la Renaissance (Tours-Paris, 27-30 juin 2017), Turnhout, Brepols, à paraître.

BALSAMO Jean, «L'italianisme lyonnais et l'illustration de la langue française », dans G. Defaux (éd.), Lyon et l'illustration de la langue française à la Renaissance, Lyon, ENS Éditions, 2003, p. 211-229.

D’AMIco Silvia et GAMBINo LoNGo Susanna (éds), Le savoir italien sous les presses lyonnaises à la Renaissance, Genève, Droz, 2017.

ZEMON DAvis Natalie, « Publisher Guillaume Rouillé, Businessman and Humanist », dans R. J. Schoeck (éd.), Editing Sisteenth Century Texts, Toronto, University of Toronto Press, 1972, p. 72-111. ZEMON DAVIS Natalie, « Le monde de l'imprimerie humaniste : Lyon », dans H.-J. Martin et R. Chartier (dir.), Histoire de l'édition française, tome 1, Le Livre conquérant : du Moyen Âge au milieu du XVII siècle, Paris, Promodis, 1983, p. 255-277.

\section{NOTES}

1. R. Gascon, «Les Italiens dans la renaissance économique lyonnaise aux $\mathrm{XVI}^{\mathrm{e}}$ siècle ", Revue des études italiennes, n. s., vol. V, 1958, p. 166-181 et Grand commerce et vie urbaine au XVI siècle. Lyon et ses marchands (environs de 1520-environs de 1580), Paris et La Haye, Mouton, 1971, 2 vol.

2. J. Boucher, Présence italienne à Lyon à la Renaissance du milieu du XV siècle à la fin $d u X V I^{e}$ siècle, Lyon, Lugd, [1994] et "Les Italiens à Lyon ", dans J. Balsamo (éd.), Passer les monts. Français en Italie - L'Italie en France (1494-1525), Paris-Fiesole, Honoré Champion-Cadmo, 1998, p. 297-312; H. Hours, « Renaissance et influence italienne ", Monuments historiques, ${ }^{\circ} 157,1988$, p. 9-11 ; J.-F. Dubost, La France italienne XVI ${ }^{e}$-XVII ${ }^{e}$ siècle, Paris, Éditions Aubier, 1997 ; G. Iacono et S. E. Furone, Les marchands banquiers florentins et l'architecture à Lyon au XVI siècle, [Paris], Publisud, 1999.

3. M. Brésard, Les Foires de Lyon aux XV et XVI siècles, Paris, Picard, 1914.

4. Pour l'histoire de l'imprimerie à Lyon à la Renaissance, voir H. L. Baudrier, Bibliographie lyonnaise. Recherches sur les imprimeurs, libraires, relieurs et fondeurs de lettres de Lyon au XVI siècle, Publiées et continuées par J. Baudrier, Lyon, Librairie ancienne d'Auguste Brun 1895-1921, 12 vol. (reprint Paris, P. de Nobèle 1964-1965), 13 vol., à mettre à jour avec S. von Gültlingen, Bibliographie des livres imprimés à Lyon au seizième siècle, Baden-Baden-Bouxwiller, V. Koerner, 1992-; M. Audin, Les Origines de l'imprimerie à Lyon et son premier siècle d'activité, La Courneuve, 
OFMI Garamont, 1973 ; J.-M. Dureau « Les premiers ateliers français », dans R. Chartier, J.-P. Vivet et H.-J. Martin (dir.), Histoire de l'édition française, t. 1, Le Livre conquérant : du Moyen Âge au milieu du XVII siècle, Paris, Promodis, 1983, p.163-175; N.Zemon Davis «Le monde de l'imprimerie humaniste : Lyon », dans ibid., p. 255-277 ; I. Andreoli, « Lyon, nom \& marque civile. Qui sème aussi des bons livres l'usage : Lyon dans le réseau éditorial européen $\left(\mathrm{xV}^{\mathrm{e}}-\mathrm{XVI}^{\mathrm{e}}\right.$ siècle) », dans J.-L. Gaulin et S. Rau (éds), Lyon vu/e d'ailleurs, 1245-1800 : échanges, compétitions et perceptions, Lyon, Presses universitaires de Lyon, 2009, p. 109-140.

5. E. Balmas, «Librai italiani a Lione ", dans A. Possenti et G. Mastrangelo (éds), Il Rinascimento a Lione, Actes du Colloque (Macerata, 6-11 mai 1985), t. I, Rome, Edizioni dell'Ateneo, 1988, p. 63-82 ; R. Scrivano, «Libri e autori italiani a Lione nel XVI secolo», dans ibid., t. II, p. 925-936; S. Albonico, «Librai italiani a Lione 1540-1580», Nuova Rivista di Letteratura italiana, vol. 3, $\mathrm{n}^{\circ} 1$, 2000, p. 203-217.

6. M. C. Misiti, «Una porta aperta sull'Europa: i de Portonariis tra Trino, Venezia e Lione. Ricerche preliminari per l'avvio degli annali», Il Bibliotecario, s. III, $\mathrm{n}^{\text {os }} 1-2$, 2008, p. 55-91; A. Nuovo, "Produzione e circolazione dei libri giuridici tra Italia e Francia (sec. XVI): la via commerciale Lione-Trino-Venezia ", dans G. P. Brizi et M. G. Tavoni (éds), Dalla pecia all'e-book. Libri per l'Università: stampa, editoria, circolazione e lettura, Actes du colloque (Bologne, 21-25 octobre 2008), Bologne, Clueb, 2009, p. 341-349; Ead., «Da Trino a Venezia a Lione. Le imprese librarie dei mercanti trinesi ", dans M. Balboni (éd), Trino e l'arte tipografica nel XVI secolo. Dal Marchesato del Monferrato all'Europa al mondo, Novara, Interlinea edizioni, 2014, p. 137-146; G. Ferraris, «Giovanni Pullone e altri stampatori trinesi a Lione», dans ibid., p. 115-136; L. Brusotto, A. Ruffino et R. Rossi (éds), Stampatori trinesi del Cinquecento. Editoria, Arte e «Avanguardia» tra Monferrato ed Europa, catalogue de l'exposition, Caluso, Grafica M. G. editore, 2013, p. 137-145 ; R. Gorris Camos, « Il torchio e la seta : la nébuleuse de imprimeurs et libraires piémontais à Lyon et leur networking franco-italien ", dans S. D'Amico et S. Gambino Longo (éds), Le savoir italien sous les presses lyonnaises à la Renaissance, Genève, Droz, 2017, p. 37-88.

7. R. Gorris Camos, Il torchio e la seta, art. cité, p. 60-61.

8. G. Heilbrun, Le premier italique en France. Balthazar de Gabiano à Lyon et les contrefaçons des éditions aldines, catalogue $\mathrm{n}^{\circ}$ 43, Paris [c. 1973] ; D. J. Shaw, «The Lyons Counterfeit of Aldus's Italic Type: A New Chronology », dans D. V. Reidy (dir.), The Italian Book, 1465-1800, Londres, The British Library, 1993, p. 117-133 ; W. Kemp, "Counterfeit Aldines and Italic-Letter. Editions Printed in Lyons 1502-1510: Early Diffusion in Italy and France ", Papers of the Bibliographical Society of Canada, Toronto, vol. 35, $\mathrm{n}^{\circ}$ 1, 1997, p. 75-100.

9. I. Andreoli, «Échanges d'images, images d'échanges: le livre illustré lyonnais à la Renaissance ", dans L. Virassamynaïken (dir.), Lyon Renaissance. Arts et Humanisme, catalogue de l'exposition (Lyon, Musée des Beaux-Arts, 2015), Lyon-Paris, MBA-Somogy, 2015, p. 62-67 : p. 62 et fig. 16.

10. J.-M. Dureau-Lapeyssonie, « Recherches sur les grandes compagnies des libraires lyonnais au $\mathrm{XVI}^{\mathrm{e}}$ siècle ", dans R. Chartier (éd), Nouvelles études lyonnaises, Genève, Droz, 1969, p. 3-77 ; W. Pettas, "The Giunti and the Book Trade in Lyon », dans A. Ganda, E. Grignani et A. Petrucciani (éds), Libri, tipografi, biblioteche, ricerche storiche dedicate a Luigi Balsamo, vol. II, Florence, Olschki, 1997, p. 169-191 ; Id., An International Renaissance Publishing Family: The Giunti, Chicago, Library Quarterly, 1974 ; Id., The Giunti of Florence, a Renaissance Printing and Publishing Family, a History of the Publishing Firm and a Catalogue of the Editions, New Castle, Oak Knoll, 2013 et L. Castelli, «I Giunta di Lione e il libro in lingua italiana: produzione, commercio, politica editoriale », dans S. D'Amico et S. Gambino Longo (éds), Le savoir italien, ouvr. cité, p. 123-152.

11. A. Morisi Guerra, «Sante Pagnini traducteur de la Bible », dans I. Backus et F. Higman (éds), Théorie et pratique de l'exégèse, Genève, Droz, 1990, p. 191-198; E. Kammerer, « Deux témoignages sur Sante Pagnini de Lucques. Les éloges de Bartholomeus Ruffus et de Symphorien Champier ", Bibliothèque d'Humanisme et Renaissance, vol. LXIV, n 3, 2002, p. 639-651; M. Engammare, "Sante 
Pagnini, traducteur ad litteram et exégète secundum allegoriam de l'écriture (1520-1536) », dans D. de Courcelles (éd.), Philologie et subjectivité, Actes de la journée d'étude (Paris, 2001), Paris, École nationale des chartes, 2002, p. 41-52 ; S. Campanini, « Pagnini, Antonio Baldino », dans Dizionario Biografico degli Italiani, vol. 80, 2015, Rome, Instituto dell'Enciclopedia Italiana - Treccani, avec bibliographie, disponible en ligne sur <www.treccani.it/enciclopedia/antonio-baldinopagnini_(Dizionario-Biografico)/>.

12. U. Rozzo, «La cultura italiana nelle edizioni lionesi di Sébastien Gryphe (1531-1541) », La Bibliofilia, vol. XC, nº 2, 1988, p. 161-195 ; R. Mouren (éd.), Quid novi ? Sébastien Gryphe, à l'occasion $d u 450^{e}$ anniversaire de sa mort, Actes du colloque (Lyon-Villeurbanne, 2006), Villeurbanne, ENSSIB, 2008.

13. Voir R. Cooper, « Gryphe préfacier », dans R. Mouren (éd.), Quid novi ?, ouvr. cité, p. 219-241.

14. U. Rozzo, «I Paradossi di Ortensio Lando tra Lione e Venezia e il loro contenuto teologico », La Bibliofilia, vol. CXIII, n 2, 2011, p. 175-209 ; S. Adorni Braccesi et S. Ragagli, «Lando, Ortensio », dans Dizionario Biografico degli Italiani, vol. 63, 2004, Rome, Instituto dell'Enciclopedia Italiana Treccani, p. 451-459, avec bibliographie; F. Greco, «Lando, Ortensio », dans Ereticopedia, 2014, disponible en ligne sur <www.ereticopedia.org/ortensio-lando>.

15. R. Weiss, «Alamanni, Luigi», dans Dizionario Biografico degli Italiani, vol. 1, 1960, Rome, Instituto dell'Enciclopedia Italiana - Treccani, p. 568-571, avec bibliographie.

16. On imprime et on publie, au sens large, près de 150 ouvrages - 80 titres - en langue italienne à Lyon au cours du XVI ${ }^{\mathrm{e}}$ siècle. N. Bingen, Philausone (1500-1660). Répertoire des ouvrages en langue italienne publiés dans les pays de langue française de 1500 à 1660, Genève, Droz, 1994 ; F. Simone, « La presenza di Dante, Petrarca e Boccaccio nel primo umanesimo lionese », dans Id., Umanesimo, Rinascimento, Barocco in Francia, Milan, Mursia, 1968, p. 59-74 ; J. Balsamo, «L'italianisme lyonnais et l'illustration de la langue française ", dans G. Defaux (éd), Lyon et l'illustration de la langue française à la Renaissance, Lyon, ENS Éditions, 2003, p. 211-229 et «Le livre italien publié à Lyon au $\mathrm{XVI}^{\mathrm{e}}$ siècle : de l'italianisme commercial à l'italianisme politique ", dans S. D'Amico et S. Gambino Longo (éds), Le savoir italien, ouvr. cité, p. 13-34 ; P. De Capitani, «Éditeurs, lecteurs et lectures de romans italiens à Lyon pendant la Renaissance ", dans ibid., p. 313-342.

17. P. Simoncelli, Fuoriuscitismo repubblicano fiorentino, 1530-1554, Milan, F. Angeli Storia, 2006.

18. Tout naturellement le premier écrivain vernaculaire contemporain à avoir une présence significative en France, et notamment à Lyon, est le représentant le plus illustre de la nouvelle dimension littéraire et non humaniste de la littérature, Pietro Aretino, dont Jean de Vauzelles traduit les œuvres religieuses : l'Umanità e la Passione di Cristo en 1539 chez Melchior et Gaspar Trechsel, puis les Sette Salmi et la Genesi chez Gryphe, respectivement en 1540 et 1542. Aretino était un « compare » du «Lyonnais » Sebastiano Serlio, encore plus que lui lié au libraire vénitien Francesco Marcolini et qui, établi à Lyon, en 1552, fera paraître chez Jean de Tournes l'Extraordinario libro di architettura en version italienne et française. Voir S. Deswarte-Rosa (dir.), Sebastiano Serlio à Lyon. Architecture \& Imprimerie, vol. 1, Le traité d'architecture de Sebastiano Serlio. Une grande entreprise éditoriale $d u X I^{e}$ siècle, Lyon, Édition Mémoire active, 2004.

19. A. Quondam, “'Mercanzia d'onore' 'Mercanzia d'utile': produzione libraria e lavoro intellettuale a Venezia nel Cinquecento », dans A. Petrucci (dir.), Libri, editori e pubblico nell'Europa moderna, Rome-Bari, Laterza, 1977, p. 51-104 ; A. Nuovo et C. Coppens, Giolito e la stampa nell'Italia del XVI secolo, Genève, Droz, 2005 ; I. Andreoli, «Giolito, Gabriele », dans M. Menato, E. Sandal et G. Zappella (éds), Dizionario degli editori e stampatori italiani del Cinquecento, vol. II, Trieste, Libreria antiquaria Drogheria 28, à paraître.

20. Voir M. Jourde, «Comment Jean de Tournes (n')est (pas) devenu un imprimeur humaniste », dans Passeurs de textes. Imprimeurs et libraires à l'âge de l'humanisme, Paris, École nationale des chartes, 2012, p. 117-131.

21. De Tournes produit 15 éditions ou émissions pour 12 titres, du Pétrarque de 1545 à l'Ethica d'Aristotile ridotta in compendio (1569) en passant par la Vita et metamorfoseo d'Ovidio (1559). Voir 
A. Cartier, Bibliographie des éditions des de Tournes imprimeurs lyonnais, Lyon-Paris, Éditions des Bibliothèques nationales de France, 1937 (Genève, Slatkine Reprints, 1970), 2 t. ; E. Kammerer, «Nouvelles hypothèses sur Jean de Vauzelles, Maurice Scève et Jean de Tournes en domaine italien », dans S. D'Amico et S. Gambino Longo (éds), Le savoir italien, ouvr. cité, p. 343-358.

22. F. Daenens, «Le traduzioni del trattato Della vera tranquillità dell'animo (1544): l'irriconoscibile Ortensio Lando ", Bibliothèque d'Humanisme et Renaissance, vol. LVI, 1994, p. 665-694 : 673-680.

23. E. Picot, Les Français italianisants au XVI siècle, Paris, Champion, 1906, vol. I, p. 183-220 ; N. Zemon Davis, «Publisher Guillaume Rouillé, Businessman and Humanist », dans R. J. Schoeck (éd.), Editing Sisteenth Century Texts, Toronto, University of Toronto Press, 1972, p. 72-111; E. Kamerrer, A. Plagnard et E. Rajchenbach-Teller, «Entre stratégies commerciales et "illustration" des vulgaires romans : la boutique de Guillaume Roville à Lyon (1548-1556) ", dans E. Kammerer et J.-D. Müller (éds), Les ateliers d'imprimeurs, lieux d'expérimentation des langues vernaculaires en Europe (fin XV'-XVI ${ }^{e}$ siècles), Genève, Droz, 2015, p. 443-487 ; E. Rajchenbach-Teller, " "De ceux qui de leur pouvoir aydent et favorisent au publiq": Guillaume Rouillé, libraire à Lyon ", dans C. Bénévent, A. Charon, I. Diu et M. Vène (éds), Passeurs de textes: imprimeurs et libraires à l'âge de l'humanisme, Genève, Droz, 2012, p. 99-116; Ead., " Mais devant tous est le Lyon marchant ». Construction littéraire d'un milieu éditorial et livres de poésie française à Lyon (1536-1551), Genève, Droz, 2015, ch. IV, p. 155-188.

24. Ridolfi était aussi l'intermédiaire pour les dédicaces aux femmes nobles passionnées par la culture italienne (Marguerite du Bourg, Marguerite de Saluces-Cardé, Madame de Termes, Maria Albizza de' Dei), et en contact avec les principaux écrivains français, dont Pontus de Tyard. Voir E. Giudici, «Luc'Antonio Ridolfi et la Renaissance franco-italienne », Quaderni di Filologia e lingue romanze, n.s., vol. I, 1985, p.111-50; R. Cooper, «Le cercle de Lucantonio Ridolfi », dans M. Clement et J. Incardona (éds), L'émergence littéraire des femmes à Lyon à la Renaissance 1520-1560, Saint-Étienne, Université Jean Monnet - Saint-Étienne, 2008, p. 29-50 ; P. Procaccioli, «Le 'tre corone' a Lione. Guillaume Rouillé e Lucantonio Ridolfi », dans S. D’Amico et S. Gambino Longo (éds), Le savoir italien, ouvr. cité, p. 223-244.

25. En effet, on retrouve encore aujourd'hui nombre de ces éditions dans les fonds anciens des bibliothèques italiennes, preuve qu'elles durent avoir une réelle diffusion commerciale en Italie.

26. N. Bingen, Le Maître italien (1510-1660), Bruxelles, Van Balberghe, 1987 ; A. Villa, « Tipologie e funzioni dei libri bilingui italo-francesi pubblicati in Francia e nello spazio francofono nel XVI secolo ", dans S. D'Amico et S. Gambino Longo (éds), Le savoir italien, ouvr. cité, p. 245-273.

27. N. Bingen, «Les éditions lyonnaises de Pétrarque dues à Jean de Tournes et à Guillaume Rouillé ", dans J. Balsamo (éd.), Les poètes français de la Renaissance et Pétrarque, Genève, Droz, 2004, p. 139-155.

28. G. Boccaccio, Decamerone, Lyon, Rouillé, p. 6-7.

29. G. du Choul, Discorso sulla religione antica de' Romani, Lyon, Rouillé, 1559, p. 3.

30. I. Andreoli, «L'Orlando furioso 'tutto ricorretto et di nuove figure adornato'. L'edizione Valgrisi (1556) nel contesto della storia editoriale edillustrativa del poema fra Italia e Francia nel Cinquecento ", dans S. Fabrizio Costa (éd.), Routes du livre ancien italien en Normandie, Actes du colloque (Caen, 2010), Berne, Peter Lang, 2011, p. 41-131; Ead., «Livres italiens à figures et "illustration" des femmes à Lyon au $\mathrm{XVI}^{\mathrm{e}}$ siècle", dans C. J. Brown et A.-M. Legaré (éds), Les femmes, la culture et les arts en Europe entre Moyen Âge et Renaissance, Turnhout, Brepols, 2016, p. 259-274 ; Ead., «Échanges d'images, images d'échanges", art. cité ; Ead., "Un "Lyonnais italianisant" : les éditions italiennes illustrées de Guillaume Rouillé », dans C. Lastraioli (éd.), "Poco a poco». L'apport de l'édition italienne dans la culture francophone, Actes du $60^{\mathrm{e}}$ colloque international d'études humanistes du Centre d'études supérieures de la Renaissance (Tours-Paris, 27-30 juin 2017), Turnhout, Brepols, à paraître.

31. J. Dubu, «Le Promptuaire des medalles de Guillaume Rouillé», dans A. Possenti et G. Mastrangelo (éds), Il Rinascimento a Lione, ouvr. cité, t. I, p. 185-219 et I. Andreoli, « La storia in 
soldoni: il Promptuaire des medalles di Guillaume Rouillé », dans M. Gabriele et U. Rozzo (éds), Storia per parole e per immagini, Actes du colloque (Cividale del Friuli, 2003), Udine, Forum, 2006, p. 235-266.

32. T. Renucci, Un aventurier des lettres $d u X^{\prime} I^{e}$ siècle, Gabriel Syméoni florentin, Paris, Didier, 1943 ; S. D’Amico et C. Magnien-Simonin (éds), Gabriele Simeoni : un Florentin en France entre princes et libraires, Actes du colloque (Chambéry, 2011), Genève, Droz, 2015; M. Barsi, "Les devises illustrées de Gabriele Simeoni. Du manuscrit Ashburnham 1376 aux éditions publiées par Guillaume Rouillé », dans S. D'Amico et S. Gambino Longo (éds), Le savoir italien, ouvr. cité, p. 461-488, et A. Parnotte, "Genèse et aventure éditoriale du Dialogo delle imprese militari et amorose de Giovio et de Le Imprese heroiche et morali de Symeoni chez Roville, 1555-1559, à la lumière du ms. Ashburnham 1376 ", dans ibid., p. 489-560.

33. I. Oriani, «Edizioni della Bibbia e dell'Iconologia di Cesare Ripa », dans L'istoriato. Libri a Stampa e Maioliche Italiane del Cinquecento, catalogue de l'exposition (Musées du Vatican, 1993), Faenza, Faenza editrice, p. 86-97; M. T. Caracciolo Arizzoli, «Les Métamorphoses d'Ovide publiées à Lyon au XVI siècle", dans J. Rosen (éd.), Majoliques européennes: reflets de l'estampe lyonnaise, $\mathrm{XVI}^{e}-\mathrm{XVII}{ }^{e}$ siècle, Dijon, Faton, 2003, p. 40-67 ; I. Andreoli, «Fabulae artificialiter pictae: illustrazione del libro e decorazione ceramica nel Rinascimento », dans M. Marini (éd), Fabulae pictae. Miti e storie nelle maioliche del Rinascimento, catalogue de l'exposition (Florence, 2012), Florence, Firenze Musei-Giunti, 2012, p. 110-125.

34. E. Barbieri, Le Bibbie italiane del Quattrocento e del Cinquecento. Storia e bibliografia ragionata delle edizioni in lingua italiana dal 1471 al 1600, Milan, Editrice Bibliografica, 1991, n. 49-50, p. 309-311; n. $53-54$, p. $316-317$; n. 60 , p. $325-329$; n. 62 , p. 331 ; n. 64, p. 335 ; n. 67, p. $341-342$; n. 69, p. 346 ; D. D’Aguanno, « Massimo Teofilo, traduttore del Nuovo Testamento », Lingua e stile. Rivista di storia della lingua italiana, $\mathrm{n}^{\circ} 1$, 2017, p. 49-86.

35. F. Gilmont, «Le "protestantisme" des libraires et typographes lyonnais (1520-1560) », Revue d'histoire ecclésiastique, vol. 101, 2007, p. 988-1013 ; U. Rozzo, « Editori e tipografi italiani operanti all'estero "religionis causa" ", dans M. Santoro (éd.), La stampa in Italia nel Cinquecento, Actes du colloque (Rome, 1989), t. 2, Roma, Bulzoni, 1992, p. 89-118 et C. Lastraioli, « Lyon 1567 ou de la diaspora des érudits et des imprimeurs italiens », dans S. D’Amico et S. Gambino Longo (éds), Le savoir italien, ouvr. cité, p. 107-122.

\section{RÉSUMÉS}

Entre le début des guerres d'Italie et la fin des guerres de Religion, qui amenèrent à terme le déclin inexorable de la prépondérance lyonnaise au sein de l'espace français du livre, de quelle importance a été, dans le Lyon de l'imprimerie, la présence italienne ? Comment ont été reçus les produits éditoriaux italiens - qu'ils soient dûs à des auteurs italiens, à des libraires italiens, ou publiés en langue italienne -, et quel rôle jouèrent certains libraires lyonnais, particulièrement tournés vers la Péninsule, dans les échanges culturels italo-français? Telles sont les trois questions auxquelles il est répondu par l'étude de groupes sociaux constitués sur une base géographique, comme les libraires piémontais ou florentins, et les modalités de leur insertion dans la vie lyonnaise, ainsi que par celle de politiques éditoriales exemplaires des «Lyonnais italianisants » : celles de Sébastien Gryphe, Jean de Tournes ou Guillaunme Rouillé. 
Tra l'inizio delle guerre d'Italia e la fine delle guerre di religione che portarono al declino inesorabile della preponderanza lionese sul mercato editoriale francese, quale importanza ha rivestito la presenza italiana nella Lione della stampa? Che diffusione hanno avuto i prodotti editoriali italiani - siano essi dovuti ad autori italiani, a librai italiani o pubblicati in italiano -,e che ruolo hanno giocato negli scambi culturali italo-francesi, alcuni librai lionesi particolarmente legati alla Penisola? Il presente contributo risponde a questi interrogativi con lo studio dei gruppi sociali costituiti su base geografica, come i librai piemontesi o fiorentini, delle modalità del loro inserimento nella vita lionese, e con quello delle politiche editoriali dei «Lyonnais italianisants» Sébastien Gryphe, Jean de Tournes e Guillaunme Rouillé.

From the beginning of the Italian wars to the end of the Religious conflicts that led to the unescapable decline of Lyon as a dominant factor in the French book trade, what was the relative weight of the Italian community in the Lyonese book world? How were received Italian cultural products-whether they were due to Italian authors, to Italian booksellers, or published in Italian -, and what part played in the French-Italian exchanges some Lyonese booksellers who were part of commercial networks with Italy? Such are the three issues that are addressed here through the study of geographically based social groups such a as the Piedmont and Florentine booksellers, and of the publishing strategies of the "Lyonnais italianisants" Sébastien Gryphe, Jean de Tournes and Guillaume Rouillé.

INDEX

Keywords : Lyon, Renaissance, book, printers, booksellers, Italy

Parole chiave : Lione, Rinascimento, libro, tipografi, librai, Italia

Mots-clés : Lyon, Renaissance, livre, imprimeurs, libraires, Italie

\section{AUTEUR}

\section{ILARIA ANDREOLI}

CNRS, ITEM Paris, Fondazione Giorgio Cini (Venise)

ilaria.andreoli@item-cnrs.fr 